\title{
Real-time PCR-based detection and quantification of genetically modified maize in processed feeds commercialised in Malaysia
}

\begin{abstract}
The present study which dealt mainly with processed feeds and some maize samples sold commercially in Malaysia evaluated the implementation of a real-time PCR cycling system for singleplex screening of eight target sequences (lectin, hmg, adh1, p35S, NK603, GA21, MON810 and MON863) and quantification of four genetically modified (GM) maize events (NK603, GA21, MON810 and MON863). The effects of using proprietary glass magnetic particles to bind DNA to their surface were also investigated in terms of DNA quantity, purity, integrity, quality and its overall effect on DNA amplification. GM material was present in $26.2 \%$ feeds and $65 \%$ maize samples. All GM samples contained MON810 followed by NK603 (47.5\%), GA21 (25\%) and MON863 (2.5\%). Single-event and multipleevents were identified in the GM samples with $50 \%$ of the GM samples containing multipleevents. The present study which represents a fast and reliable methodology would provide an overview of the presence and levels of GMOs in feeds and maize in Malaysia.
\end{abstract}

Keyword: DNA; GMO; GMO quantification;Feeds; Real-time PCR 